DOI: $10.15826 /$ qr.2015.1.079

УДК 94(470)“16/18”

$+94(44) “ 16 / 18$ " +

$272 / 273+271.2$

Francine-Dominique Liechtenhan

\title{
HENRY LA VIE, SPION, KONSUL UND VEREHRER PETERS DES GROSSEN
}

Henry La Vie, marine commissioner and consul of France in Saint Petersburg in the 1710s-1720s, left an exceptional correspondence in Russia at the time of Peter the Great. He was involved in the establishment of diplomatic and economic relations between France and Russia, but, for no apparent reason, Versailles did not trust him. Many of his clever suggestions were not accepted by the Regent. Responsible for a small French community in Saint Petersburg, he studied the relationship between Catholicism and Orthodoxy, and condemned the harmful role of Jesuits.

After the suspicious death of Alexei Petrovich, La Vie made a lot of speculations about the future of Russia and the change of international relations, particularly in the North. Versailles was not able to duly appreciate the letters of La Vie at their real value; his debts were an easy pretext to remove him from his important position. He disappeared in Russia and died probably in the late 1730s. His best letters from Russia should be selected and prepared for a publication.

Keywords: Henry La Vie, the time of Peter the Great, relations between France and Russia, Catholicism and Orthodoxy.

Анри Лави, служивший уполномоченным по морским делам и консулом Франции в Санкт-Петербурге в период между 1710-1720-ми гг., является автором значительного числа писем, датируемых эпохой Петра Великого. Он участвовал в установлении дипломатических и экономических отношений между Францией и Россией, однако не пользовался доверием Версаля, поэтому многие его рациональные предложения не находили поддержки у регента. Возглавляя небольшое французское сообщество в Санкт-Петербурге, он изучал отношения между католицизмом и православием и говорил о губительном влиянии иезуитства. После загадочной смерти Алексея Петровича Лави много рассуждал о будущем России и изменениях в международных отношениях, особенно на севере. Версаль не признал важности писем ла Ви и под предлогом долгов сместил его с высокой должности. След его теряется в России, где он умер, предположительно, в 1730-х гг. Его лучшие письма заслуживают публикации.

Ключевые слова: Анри Лави; эпоха Петра Великого; отношения между Францией и Россией; католицизм и православие.

(C) Liechtenhan F-D., 2015

Quaestio Rossica • 2015 • №1, p. 59-70 
„In Abwesenheit des Zaren sind die Nachrichten undurchschaubar, aber die verschiedenen Parteien lassen es sich nicht nehmen, sich über die jetzige Konjunktur zu äussern", dieser Satz, der wiederholt in der Korrespondenz des französischen Konsuls La Vies vorkommt, ist für die Meinung des Autors bezeichnend. Er bewunderte den Zaren, seinen Helden, blieb jedoch oft widersprüchlich in seinen Überlegungen zur russischen Außenpolitik. Er sah sie vor allem durch das Guckloch der politischen, wirtschaftlichen und religiösen Beziehungen zum Westen; hin und wieder ergötzte er sich an Skandalgeschichten, Klatsch, aber zog aus ihnen wichtige Schlüsse. Es fehlte ihm nicht an diplomatischem Spitzengefühl.

\section{Der erste Vertreter Frankreichs in Petersburg}

Henry La Vie wurde 1678 in Bordeaux als Sohn eines protestantischen Händlers britischer Abstammung geboren [Mézin, Rjéoutski; Augereau; AE, B1, 983 (1720-1724), fol. 51-52 (Brief vom 10. Mai 1720 an den Regenten)]. Die ganze Familie liess sich 1685 zum Katholizismus bekehren. La Vie war ein Abenteurer, arbeitete zeitweilig für die französische Krone, musste jedoch, total verschuldet, mehrere Male das Land verlassen. 1710, floh er nach Venedig, wo er den baron Urbich, den Representanten des Zaren, kennenlernte. Er trat in dessen Dienste und folgte ihm als Sekretär nach Regensburg und Wien. Ein Jahr später findet man ihn in Hamburg, wo er sich dem franzöischen Konsul Abensur näherte und in eine Spionagegeschichte verwickelt wurde. Er übergab ihm mehrere Texte über Frankreichs Interesse, einen direkten Handel mit Russland zu betreiben. In der Zeit wurden die Waren - aus Russland Rohmaterialien, aus Frankreich Luxusgüter und manufakturierte Produkte - ausschließlich mittels britischer oder holländischer Frachtkompanien verschickt, was mit zusätzlichen Kosten verbunden war. La Vie setzte sich schon in seinen frühesten Schriften für einen direkten, bilateralen Handel ein (Berichte vom 19. Juli 1712, aus Hamburg [AN, Marine, B7, 15, fol. 163]; vom 7. November 1712, „Négociations entre l'empereur et le tsar. Moyens d'attirer le tsar vers la France <Traité de commerce éventuel> " [B7, 16, fol. 201-203 B7, 18, fol. 47-59; vom 4. April 1713, „Projet de traité de commerce avec la Moscovie" [B7, 18, fol. 47-59]). Wir finden ihn 1713 in London wieder, wo er an der Gründung einer Grossrussischen Handelskompanie beteiligt war. Diesmal schaffte er es, das Interesse Pontchartrains zu wecken; der französische Marineminister träumte seit langem davon, dass die wenigen franzöischen Händler in Russland die gleichen Privilegien wie die Briten oder Holländer geniessen dürften. Ende des Jahres entstand eine „Compagnie de Saint-Pétersbourg“ deren Grundkapital sich über die $500000 £$ erhob. Eine wichtige Summe, die La Vie zum Teil selbst investierte; woher er das Geld hatte, ist unklar, vielleicht hatte er Familiengüter in England verkauft. Vier erste Schiffe sollten von Rouen aus diesen Handel ins Rollen bringen (Bericht vom 
23. November 1713 aus Rouen [AN, AE, B1, 982, fol. 19-23]). La Vie verlangte als Belohnung die Stelle des französischen Konsuls in Archangelsk; damals war die Stadt noch der wichtigste Hafen Russlands. Wenig später wollte er den Petersburger Posten, der allerdings noch gegründet werden musste, denn Frankreich hatte keine Representation in Russland. La Vie war also von Peters Willen, aus der Stadt den ersten Hafen seines Reiches zu machen, wohl informiert (Brief vom 9. April 1713 [AN, AE, B1, 982, fol. 1-4]). Auch wenn Pontchartrain ihn sehr unterstützte, war Versailles diesem Abenteurer gegenüber misstrauisch, und das Kabinet von diesen französisch-russischen Projekten wenig überzeugt. La Vie wurde schliesslich zum Marinekommissar in Petersburg ernannt und erhielt eine Pauschalsumme von $18000 £$, allerdings ohne regelmässigen Lohn. Er musste also für sich selbst aufkommen und mit den mageren Zolleinnahmen auskommen. Frau und Kinder blieben in Bordeaux. Die Wahl dieses undurchsichtigen Mannes, die Bedingungen, die ihm auferlegt wurden, zeugen wie wenig man von einer diplomatischen Beziehung mit Russland überzeugt war. Frankreich war mit dem spanischen Erbfolgekrieg beschäftigt, sein Hauptaugenmerk lag auf Österreich und Grossbritannien [Bély, S. 525 sq.]. Der Osten Europas war in der Logik der „Barrière de l'Est“, der Ostbarrière, festgefahren, die vorsah, dass Polen, Schweden und die Pforte, Frankreichs Verbündete, die barbarischen Moskoviter in Schranken halten sollten und vor allem eine Annäherung zwischen Wien und Petersburg verhindern sollten. Dass man nach dem Sieg von Poltava und nach den ersten Reformen Peters "nicht mehr mit Moscovitern, sondern mit Russen“ zu tun hatte, daran dachte das Cabinet nicht (Brief vom 6. November 1716 aus Pétersburg an Jean d'Estrées (1666-1718, Theologe, Diplomat un Staatsrat) [AN, AE, B1, 982, fol. 146-148]). La Vies zahlreiche Briefe an seine Vorgesetzten versuchen verzweifelt dieses Vorurteil zu bekämpfen und die Fortschritte Russlands darzustellen. Bezeichnend ist, dass Versailles den Tod Ludwig XIV. an alle Höfe vermittelte, aber den Zaren vergass; Schafirov wurde beauftragt, dessen Unwillen in neutralen Worten auszudrücken. Völlig eingeschüchtert sah sich La Vie genötigt, keine Trauerkleider anzuziehen bevor die Notification in Petersburg eintraf (Brief vom 20. Oktober 1715 an den Conte de Toulouse) (Louis Alexandre de Bourbon, natürlicher Sohn Ludwig XIV., mit fünf Jahren Admiral der französischen Flotte, später Chef des Marinerates) [AN, AE, B1, 982, fol. 70-71]).

Die Instruktionen des Aussenministeriums waren entsprechend karg. La Vie sollte den Handel zwischen Russen, Briten und Holländern bespitzeln, deren Quantität und Qualität ermessen und vor allem die Importtarife und die Verkaufstarife der Spirituosen herausbekommen. Versailles roch immerhin einen guten Absatzmarkt für Frankreichs Weine. La Vie sollte auch den Wechselkurs zwischen Rubel und britischer oder holländischer Währung studieren. Seine wichtigste Aufgabe war es jedoch, sich um die in Russland ansassigen Franzosen zu kümmern; um 1714 konnte man sie auf ein paar Händler und hugenotische Emigranten 
reduzieren; nach Peters Paris-Aufenthalt (1717) sollte die kleine Kolonie auf etwa tausend Köpfe wachsen, die sich alles andere als durch gutes Benehmen zur Schau stellten. In La Vies Korrespondenz findet man unzählige Korruptionsfälle, Mordgeschichten und andere sittliche Skandale. Die Originalfassung der Instruktionen ist nicht unterzeichnet, es geht sehr wahrscheinlich um Pontchartrain, denn es heisst weiter, in Anbetracht, dass „die Person“, die nach Petersburg geschickt würde, „zu Höherem fähig sei“, könne sie die Persönlichkeit, „le génie“, des Zaren studieren, sowie den Charakter seines Nachfolgers, Alexey Petrovitchs. La Vie hatte auch die Aufgabe, Menchikov und die wichtigsten Minister und Generäle zu beobachten. Er sollte auch die Interessen des russischen Hofes, dessen Bezug zu anderen Staaten untersuchen. Wichtig schien auch, dass er die russische Expansionspolitik, die sich gegen die Verbündeten Frankreichs richtete, einschätzen könne. Dazu kamen die klassischen Untersuchungen, wie die Einnahmen der Krone, die Zahl der Rekruten, die Rolle des Klerus, usw [Rambaud].

La Vie hat im Rahmen seiner Möglichkeiten sein Bestes getan; aber er hatte keine Akkreditierung und einen unwichtigen Status (Brief vom 22. Februar 1715 [AN, AE, B1, 982, fol. 40-41]). Er konnte also nur bedingt die Hofaffairen verfolgen. Weit mehr beengend war noch, dass er keine Chiffren bekam, um geheime Mitteilungen zu schicken. Seine gesammte Korrespondenz, immerhin ein paar hundert Briefe, konnte also von der russischen Zensur gelesen werden. Er bemühte sich um so mehr um einen neutralen Stil. Seine Stellung blieb ambivalent: ohne Akkreditierung war er eine Privatperson, und trotzdem war er von seiner Regierung offiziel beauftragt worden, in Russland zu weilen. 1720 klagte er, keine Garden als Begleitung zu haben und immer weniger offizielle Enladungen zu bekommen; sei das nicht ein mangelnder Respekt gegenüber Ludwig? Der russische Hof würde seine missliche Lage falsch interpretieren und durch seine Person Frankreich nicht gebührend achten (Brief vom 19. August 1720 an Dubois [AN, AE, B1, 982, fol. 67-70]). Polyglott - er lernte sogar ein bisschen russisch - schaffte er es trotzdem, gewisse Persönlichkeiten näher kennenzulernen; er befreundete sich mit dem Leibarzt Areskin und pflegte regelmässigen Kontakt zu Shafirov, Golovkin, Matveev, Petr A. Tolstoj, Savva Raguzhinskij aber auch zum Hanoveraner Weber und Jean Lefort , einem Neffen François' und Vetrauten Menchikovs. Obwohl schwer verschuldet, zögerte La Vie nicht, den russischen Ministeriumsmitgliedern Geld für Informationen in die Tasche zu stecken. Vor Peters enttäuschender Frankreichfahrt konnte er den Zaren mehrere Male sehen und sogar mit ihm sprechen (Brief an Pontchartrain vom 7. Februar 1715 [AN, Marine, B7, 25, fol. 180]). Er blieb zehn Jahre lang im Amt, wurde im November 1717 sogar Konsul, musste aber noch zwei Jahre auf die notwendigen Chiffren warten. La Vie schrieb eine grosse Anzahl Memoiren über den russischen Handel; seine Korrespondenz, die im Archiv des französischen Aussenministeriums und im pariser Staatsarchiv aufgehoben ist, ist reich an Informationen über das damalige Russland. 


\section{Erste diplomatische Schritte}

La Vie kam also am 13. Januar 1714 in Petersburg an, ziemlich genau vier Monate vor dem Utrechter Friedenskongress. Unser Marinekommissar stellte sogleich einen Bezug zwischen dem Nordischen Krieg und dem spanischen Erbfolgekrieg her. Könnte Peter nicht den Kampf mit dem natürlichen Allierten Frankreichs, also Schweden, momentan aufgeben, um Druck auf Österreich auszuüben? Wie wärs auch, wenn Russland die Bourbonische Erbfolge in Madrid anerkennen würde? Beide Fragen ernteten das Missverständnis seiner russischen Gesprächspartner, vor allem Schafirovs; das siegreiche Russland würde den Kampfplatz nicht verlassen, um seinen österreichischen Verbündeten zu bekriegen. Was Spanien betraf, war der Austausch von Diplomaten in Betracht zu ziehen, doch sollte Madrid zuerst die Nofikation der Ernennung des Bourbonen nach Russland schicken und die Beziehungen unilateral ankurbeln. Russland war nicht bereit, sich direkt in diesen Konflikt einzumischen (Brief an Huxelles vom 19. August, 1715 [AN, Marine, B7, 27, fol. 4]). In Frankreich lauschte man auf La Vies Worte, das Kabinet hegte schon länger den Gedanken, eine eventuelle Vermittlerrolle im Nordischen Krieg zu übernehmen, um Druck auf Österreich auszuüben. Doch wurde mehrere Jahre lang nichts unternommen; als Frankreich die Mediation im Norden übernahm, war die spanische Erbfolge längst abgeklärt. Sobald es La Vie nur möglich war, bespitzelte er die österreichischen Vertreter, vor allem Pleyer; verärgert notierte er, dass er bei Festessen meistens gegenüber dem Zaren sass und somit in Russland eine privilegierte Rolle spielte. Mardefeld war ihm ebenso ein Dorn im Auge und er zweifelte sehr an eine dauernde preussisch-russische Beziehung, und schon gar nicht in Kombination zu Frankreich. La Vie sah die Spannungen zwischen Regenten und Zaren voraus. Peters Reise nach Frankreich im Sommer 1717 war kein politischer Treffer; Kardinal Dubois zog eine englische Allianz vor, und es kam nur ein belangloser Wirtschaftsvertrag zu Stande. Seinen einzigen wichtigen Erfolg erkor Peter in der Akademie; er wurde zum ausserordentlichen Mitglied ernannt und konnte einen reellen Austausch mit der französischen Institution herstellen, auch wenn die Petersburger Akademie nicht nach deren Modell aufgebaut wurde [Demeulenaere-Douyère]. La Vie hatte sich sehr eingesetzt, um die Kontakte mit dem Abt Bignon, dem Begründer der königlichen Bibliothek, der heutigen französischen Nazionalbibliothek, und anderen Akademiemitgliedern herzustellen; in dieser Zeit hat er sich auch Huyssen genähert und dessen Korrespondenz mit der Akademie sehr gefördert [Liechtenhan, 2010]. Auch nach Peters Rückkehr, unterstützte der frischernannte Konsul den wissenschaftlichen Austausch; so kümmert er sich um ein Zebra, das nach Russland verfrachtet werden sollte, oder um den Versand tibetanischer Manuskripte, die in Frankreich übersetzt werden mussten. Es fragt sich, ob La Vie diese wissenschaftliche Seite des internationales Austauschs wirklich voll verstand und auszunutzen versuchte, oder ob er nur der Konjunktur folgte. 


\section{Religiöse Intrigen}

La Vie musste sich um seine katholischen Glaubensbrüder kümmern und kam mit den Jesuiten in Kontakt. Er war ein überzeugter Anhänger von Peters Toleranzpolitik (Brief vom 1. April 1715 aus Petersburg [AN, Marine, B7, 26, fol. 69-83]), zeigte sich aber diesem Orden gegenüber besonders misstrauisch. Er beobachtete dessen Machenschaften in der unmittelbaren Umgebung des Zaren. Laut Matveev würden die Anhänger Loyolas sich immer wieder in die russischen Staatsgeschäfte einmischen und hätten einen starken Einfluss auf die nähere Umgebung Peters (Brief vom 27. Mai 1717 an Huxelles [AN, AE, B1, 982, fol. 185-186]). Verärgert schrieb er mehrere Male, dass sie wegen ihrer Treue zum Heiligen Stuhl Peters Frankreichfahrt sabotiert hätten. In Paris war der Zar mit den romabtrünnigen Jansenisten, insbesondere mit dem Kardinal de Noailles in Kontakt gekommen; man munkelte, dass der russische Monarch sich zu deren Religion bekehren wolle. Dieser verstand schnell, dass die Brüder noch immer von einer Vereinigung der Ostkirche mit Rom träumten und seinen Namen zu Propagandazwecken missbrauchen würden [Pleyer; Mémoires; Liechtenhan, 2011]. Er wimmelte sie sarkastisch ab, er sei Soldat und habe sich nicht um religiöse Fragen zu kümmern. La Vie sah in Österreich den politischen Feind der französisch-russischen Annäherung. Er vermutete hinter den Intrigen der Jesuiten die Hand des Kaisers, der Frankreich isolieren wolle, weil es Distanz zum Papst genommen hatte und „heretische Alianzen“, etwa mit England oder Preußen, unterzeichnen könne. La Vie beobachtete auch mit viel Misstrauen deren rege Tätigkeit in China; die Missionen würden der katholischen Kirche in Russland ein Vermögen kosten. Sie habe anderes zu tun, so zum Besipiel den vielen Franzosen zu helfen, die dem Ruf Peters, sich in Russland niederzusetzen, gefolgt waren und elend gescheitert waren. La Vie warnte jedoch das Kabinet, die Vertreibung der Jesuiten als politischen Akt Peters gegen den Kaiser zu deuten; er schrieb es dem personlichen Überdruss des Zaren zu. Er untersuchte den Ukas sprachlich und bewies, dass er im Gegenteil sehr vorsichtig und mit diplomatischem Takt geschrieben worden war (Brief La Vies an Dubois vom 4 (16) mai 1719 [SIRIO, Bd. 15, S. 27]).

\section{Überlegungen zur Thronfolgefrage}

Für La Vie waren die religiösen Fragen die Achillesferse Russlands. Peter war sein Held, er hatte Russland geschaffen; aber wie lange konnte diese neuentstandene Nazion überleben? Die Landbevölkerung war jederzeit zu Aufständen bereit, die entwder von „obskuren und betrunkenen Priestern“ oder infolge der schweren Steuerlast entfacht wurden. Das Volk verstand den ewigdauernden Krieg nicht und noch weniger die Reformen des Zaren. Am Hof und in der Regierung waren Zwistigkeiten nicht selten und La Vie nennt klar Dolgoruki, président des Senats, als Anführer verschiedener 
Verschwörungen gegen Menchikov (Brief an Huxelles vom 11. Februar 1715 [AN, AE, B1, 982, fol. 40-41]). Diesen Aufkömmling nannte er den „Sockel des Imperiums“, weil er den Wandel Russlands verkörperte. Peter konnte auf ihn zählen, weil dieser Arrivist ohne ihn nie den höchsten Rang der Gesellschaft erreicht hätte und längst den Hofintrigen erlegen wäre.

Peter soll die Flucht Alexeys ausschliesslich Menchikov zugeschrieben haben; es soll ein Protestakt gegen dessen ehemaligen Erzieher gewesen sein. Als der Tsarevitch in Neapel weilte, soll dieser gesagt haben, dass er nur zurückkomme, wenn Menchikov verbannt würde. $\mathrm{Zu}$ spat habe der Zar begriffen, dass es ein abgekartertes Spiel gegen ihn selbst war. Alexeys Aufenthalt in Wien wird ausgiebig kommentiert. Mit Genugtuung teilte der frischernannte Konsul mit, dass Peter „ressentiments“ gegen den Kaiser habe, weil er seinem Sohn in Wien „seine protection“ genehmigt hätte. Er würde sich bei nächster Gelegenheit rächen. Menchikov habe darauf geantwortet, dass die österreichische Armee sehr siegreich gegen die Türken gewesen sei und dass erneute Spannungen mit dem Wiener Hof gefährlich seien. Er habe ihn auch an den Verwandschaftsgrad zwischen Kaiser und russischem Prinzen erinnert (dank dessen Ehe mit einer Prinzessin von Braunschweig-Wolfenbüttel), der die Haltung Wiens erklären würde. La Vie anerkannte also eine gewisse Intelligenz und Klarsichtigkeit Menchikovs. Wütend habe der Zar seinen Schützling gegen die Wand gestossen und soll geschrien haben: „Ihr gehört also zu seinen Partisanen“ (Brief vom 14. Dezember 1717 an Huxelles [AN, AE, B1, 982, fol. 222-223]). Weinend habe Menchikov ihn darauf um Verzeihung gebeten!

In mehreren Briefen geht La Vie auf Russlands juristisches System ein. Es gäbe niemanden, der die Justitz verwalten würde. In diesem Punkt verurteilte der Franzose den Zaren. Wie konnte er Adelige, sogar aus Boyarengeschlecht stammende Männer durch einfache Offiziere richten lassen, die das Zivilrecht nicht kannten und meistens kaum lesen und schreiben konnten? Petersburg wäre daran eine unheilvolle, melancholische Stadt, zu werden, wegen der vielen Anschuldigungen und Prozessen um Alexey, die wie eine öffentliche allgemeine Ansteckung grassieren würden (Brief vom 1. Januar 1720 an Huxelles [AN, AE, B1, 983, fol. 34]). Alleine der „Heilige Name“ des Zaren würde so viel Angst einflössen, dass jeder seinen Groll verschweigen und ihm heuchlerisch huldigen würde. Der Zar habe somit seine Eliten gegen sich aufgebracht. Die „Majestät“ seines Imperiums sei am zerbröckeln; schon 1718, war La Vie überzeugt, der Prinz wolle das Werk seines Vaters zerstören. Die vielen Ausländer, die in russischen Diensten standen und Schlüsselstellungen am Hof und in der Regierung hatten, glaubten, dass Peters Tod Ihren Untergang bedeuten würde, denn das Volk, das im Elend siechte, würde sich sofort an ihnen rächen. Der Konsul berichtete von zahlreichen Selbstmorden in den russischen und ausländischen Eliten (Brief vom 20. Oktober 1719 an den Regenten, Marine [B7, 39, fol. 173]). Wer würde sich, unter diesen Umständen, um den letzten Willen des Zaren kümmern und Peter Petrovitch, seinen fünfährigen Sohn aus zweiter Ehe, gegen 
den Erbfolger Alexey verteidigen, der viele Anhänger hatte und zweifellos vom Kaiser unterstützt würde? Für La Vie, stürzte sich Peter mitsamt seines Landes durch diese Alexey-Geschichte in den Abgrund (Brief vom 21. Februar 1718 an Huxelles [AN, AE, B1, 982, fol. 231]). Die Rückkehr des Thronfolgers kündigte eine neue Terrorwelle an; der Zar suchte mit allen Mitteln die Organisatoren seiner Flucht. Komplizen sollten durch Tortur zum sprechen gebracht werden. Alle befürchteten, dass der junge Mann in ein Kloster gesteckt werden würde, was den keimenden Bürgerkrieg nur verschieben würde (Brief vom 23. Dezember 1718 an Huxelles [AN, AE, B1, 982, fol. 271-272]). Es gab nur eine schwache Hoffnung: die Garde. Vor allem das Preobrajenski-Regiment würde dem Zaren bis zuletzt zur Seite stehen.

La Vie stellte einen Bezug zwischen der Geschichte des Tsarevitschs und den politischen Beziehungen zum Kaiserreich. Er hegte an Alexeys Mord, auch wenn er ab und zu von Krankheit sprach, keinen Zweifel, verurteilte ihn jedoch nicht ; für ihn waren sowohl Pleyer (ab 1718 Resident) als auch die Jesuiten indirekt schuld daran. Freudig leitete La Vie die wildesten Gerüchte weiter; der Wiener Hof habe ein Protestschreiben gegen Tolstoj verfasst. Dieser sei der Urheber von Alexeys Österreich-Aufenthalt und habe sogar vom Kaiser Geld für den Aufenthalt bekommen. Er sei aber beleidigend gewesen, indem er Colloredo jeglichen Kontakt zum Prinzen untersagte hätte. Der Kaiser soll seinen Unmut, seinen Verwandten nicht sehen zu dürfen, kaum verborgen haben. Tolstoj soll unverschämt geworden sein, als die Wiener Behörden die Abreise vertagten; er habe sogar mit einem militarischen Eingriff des Zaren gedroht! La Vie blieb diesen Interpretationen gegenüber realistisch; der Zar brauche Frieden mit Österreich, ein Grund mehr Alexey von der politischen Szene zu schieben (Briefe vom 31. Januar 1718 [AN, AE, B1, 982, fol. 224-225] und 4. März 1718 an Huxelles, Marine [B7, 35, fol. 102]). Er erzählte noch viel unglaublichere Geschichten; Peter wolle seinen Sohn zu Patriarchen erheben. Pragmatisch kommentierte La Vie, der Zar müsse ihn in diesem Fall Vater nennen und ihm die Hand küssen, eine Unterwerfung des Staates gegenüber der Kirche, die nicht denkbar war [Ibid.]. Der Tod Alexeys war also eine Frage der Staatsraison, es ging um die Sicherheit des Zaren und seines Werkes; man könne nur die bewundernswerte Haltung des Monarchen loben.

\section{Das Ende einer Dynastie?}

Der Skandal isolierte jedoch Peter. La Vie beobachtete, dass der Zar sich seltener in der Öffentlichkeit zeigte und vermehrt die Quellen von Olmütz besuchte. Niemand würde es jetzt wagen, ihm die Gefahren seiner Politik zu erläutern; selbst Menchikov würde solchen Fragen aus dem Weg gehen. Die Minister würden alle Peter ihre Karriere verdanken und würden ihm immer Recht geben (Brief vom 22. September 1719 [AN, Marine, B7, 
39, fol. 125]). Angst und Fatalismus seien des ersten russischen Kaisers tägliches Los; er würde psychosomatisch einer Krankheit zum Opfer fallen (Brief an Dubois vom 26. September 1718 [AN, AE, B1, 982, fol. 252]). Vermehrt ging La Vie in den letzten Jahren auf die Nachfolge Peters und deren Folgen auf die russische Aussenpolitik ein. Schon im Juli 1719 wurde von einer eventuellen Ehe zwischen dessen zehnjährigen Tochter Elisabeth und Ludwig XV geredet. La Vie schenkte diéser Nachricht keinen Glauben. Wichtiger schien ihm die Hochzeit des älteren Kindes, Anna, mit einem Holsteiner. Er erwähnte sie oft als potentielle Nachfolgerin ihres Vaters, was bedeuten würde, dass die russische Krone nach der Hochzeit automatisch in deutsche Hände geraten würde. Dem französischen Vertreter kam es nicht in den Sinn, dass eine Frau die Regierungsgeschäfte erledigen könne, da die Frauen in Frankreich von der Thronfolge ausgeschlossen waren. Er sah Anna entweder als Regentin bis zur Volljährigkeit ihres (noch nicht geborenen) Sohnes oder als Vertreterin der Romanovs, allerdings unter der Vormundschaft ihres zukünftigen Gatten. Jedenfalls würde dies eine Annäherung mit Schweden bedeuten, dessen Erbe ebenso ein Holsteiner war, was Frankreichs Position im Norden gefärden konnte. Die andere Variante war eine Nachfolge durch den Enkelsohn des Zaren, Peter Alexeevitch, ein Braunschweiger mütterlicherseits, was eine Annäherung zwischen Russland und Österreich nach sich ziehen würde. Das Kind wurde von Peter abgöttisch geliebt, da es schon früh militärische Begabung zeigte. Eine Gefahr mehr für Versailles! La Vies geopolitisch-strategische Analysen werden Ende 1718 immer interessanter. Er kommentierte diese unzähligen Gerüchte als die Krise im Norden sich wieder zuspitzte. Peter weigerte sich am Braunschweiger Kongress teilzunehmen und zog es vor den militarischen Druck auf Schweden zu verstärken (Brief La Vies vom 1. Januar 1720 [AN, AE, B1 983, fol. 3-4]). Der Konsul beschrieb seitenlang, welche Bataillone freigestellt wurden und wie sogar Hilfstruppen schon im Anmarsch seien (Brief La Vies ans Kabinet vom vom 11. Januar 1720 [AN, AE, B1, 983, fol. 10] und vom 24. Januar 1720 [fol. 13-15]). Es war, schrieb La Vie nachdrücklich, höchste Zeit, dass Frankreich die Vermittlerrolle übernahm. Die Lage schien ihm umso brenzliger, dass ja in Wien die Erbfolge auch nicht klar bestimmt war (Brief vom 11. Januar 1720 an den Regenten [AN, AE, B1, 983, fol. 6-7]). Kurfürsten konnten die Nachfolge für sich beanspruchen und das Reich destabilisieren. Dessen Nachbarn konnten militarisch eingreifen und sich Territorien aneignen. Um die Ruhe in Europe zu wahren, musste der Regent endlich reagieren!

La Vie konnte sich nicht enthalten, Peters neue Kriegsvorbereitungen darzustellen; er wollte sensationnelle Berichte verschicken, um seine eigene Stelle zu wahren und in der Karriere weiter zu kommen (Brief ans Ministerium vom 8. Februar 1720 [AN, AE, B1, 983, fol. 17]). Er insistierte, Peter wolle vor allem seine eigenen Eroberungen sichern (Brief vom 27. Mai 1720 an den Marinerat [AN, AE, B1, 983, fol. 62-63]); falsche Friedensberichte würden ihn nicht irreführen. Peter handle mit Umsicht um nicht betrogen zu werden (Brief vom 27. Dezember 1720 an den 
Marinerat [AN, AE, B1, 983, fol. 95] und 3. März 1721 [AN, AE, B1, 983, fol. 101]). Der Konsul zeigte sich überzeugt, dass der Zar alles daransetzen würde, einen Holsteiner in Stockholm auf den Thron zu erheben und in diesem Sinne konnte er auf Karl VI. zählen. Der Kaiser hatte von Russlands Siegen profitiert, um die Schweden aus den deutschen Territorien zu vertreiben. Ein deutscher Prinz stellte eine Garantie für diese territorialen Errungenschaften dar. Für La Vie hatte der Kaiser allen Grund, sich vor der Präsenz des englischen Königs in Deutschland (dank der Verbindung zu Hanover) zu fürchten, ebenso vor dem Aufstieg Preußens, die beide nach Petersburg blickten; er habe also grösstes Interesse sich mit dem Zaren zu verbinden und Ehen zwischen Russen und Deutschen zu fördern (Brief vom 27. Mai 1720 an den Marinerat [AN, AE, B1, 983, fol. 62-63]).

Nicht weniger umsichtig kommentierte er die Vermittlung Frankreichs im Nordischen Krieg; Gerüchten zufolge, habe Versailles beschlossen, den Zaren mit Vorschlägen zu „amusieren“, d.h. ihn abzulenken, um seine Beziehung zu Stockholm zu betrüben, aber auch um Zeit zu gewinnen. Alles solle darangesetzt werden, die russische Deutschlandpolitik durch die Präsenz des Vermittlers Campredon zu schwächen. Allerdings war Versailles immer noch nicht bereit, mit Russland diplomatische Beziehungen zu knüpfen oder eine Allianz zu schließen. La Vie verstand schnell, dass der Zar seine Nachfolge als Politikum in die Wagschale legte. Wenn man seiner Logik folgt, konnte Peter kaum einen Erben ernennen, denn er hätte seine Position auf dem europäischen Schauplatz verloren. Eine Theorie, die nicht uninteressant ist. Brauchte er wirklich die Vermittlung Ludwigs, dessen Neigung zu Schweden bekannt war? Russen und Schweden konnten ohne weiteres direkt zu einem Abkommen finden. Auch in Stockholm war die Nachfolge unsicher, und es kam eigentlich nur ein Holsteiner in Frage. Sollte Anna ein Familienmitglied heiraten und eine ensprchende Aussteuer bekommen (es ging um 2 millionen Rubel) würde Russland einen direkten Zugang zur schwedischen Regierung bekommen und auf diese mittels der Rückeroberung der Städte Bremen und Werden einen kolossalen Druck ausüben. Der Kongress von Nystadt war somit für La Vie ein Scherz, der zu keinen (für Schweden und Frankreich) befriedigenden Lösungen führen könne, da der Zar in einer absoluten Machtstellung war (Brief vom 3. März 1721 an den Marinerat [AN, AE, B1, 983, fol. 101-102]). Mit diesen Spekulationen sah der kleine, ungeübte Konsul, die ganze Aussenpolitik Russlands im XVIII. Jahrhundert voraus.

\section{Der Undank Versailles}

La Vie hatte sich allerdings mit seinen vielen, zum Teil widersprüchlichen Spekulationen über eine mögliche französische Vermittlung selbst eine Grube gegraben. Als der Geschäftsträger Campredon in Petersburg auftauchte, sah er im Konsul einen gefährlichen Rivalen ; er beschuldigte den schwer verschuldeten La Vie ein englischer Spion zu sein, schlimmer noch, ein gefährlicher Calvinist! Er hielt ihm seinen freizügigen 
Lebenswandel vor; sein Haus war mehr oder weniger als Spielgrube und Bordell bekannt (Brief Campredons ans Ministerium vom 19. Juli 1723 [AN, AE, B1, 983, fol. 167]). Am 4. Januar 1722 wurde la Vie befohlen, nach Frankreich zurückzukehren [Mézin, S. XXXI]. Zwei jahre später weilte er immer noch in Petersburg. Um die Ehre Frankreichs zu wahren, versprach das Kabinett seine Schulden, immerhin $40000 \mathfrak{E}$, zu bezahlen. Nichts geschah, und Campredon musste zur Tasche greiffen (er lieh ihm $2000 £$ ), während La Vie sich weiter verschuldete. Anfang 1726 verschwand unser Mann; es wurde gemunkelt, er sei als Privatlehrer untergekommen. Er soll 1738 gestorben sein, aber wir haben keine handfesten Beweise zu seinem weiteren Schickal.

\section{Список литературы}

Лиштенан Ф.-Д. «Если Папа считает себя непогрешимым, то он, должно быть, глупец, а если не считает, то мошенник» (Петр Великий) / пер. с фр. И. Д. Гузевич, Д. Ю. Гузевича // Культурные инициативы Петра Великого: материалы II Междунар. конгресса петровских городов, Санкт-Петербург, 9-11 июня 2010 года. СПб. : Европейский дом, 2011. С. 58-62.

Ожеро К. Учреждение первого консульства Франции в Санкт-Петербурге в 1717 году / пер. Н. П. Таньшиной // Россия и Франция. XVIII-XX века. Вып. 8 / отв. ред. П. П. Черкасов. М. : Наука, 2008. С. 9-30.

Bély L. L'Art de la paix en Europe, naissance de la diplomatie moderne, XVIe-XVIIIe siècles. Paris : PUF, 2007. P. 525 sq.

Demeulenaere-Douyère C. L'Académie des Sciences de Paris ; un lieu privilégié des échanges scientifiques entre la France et la Russie (XVIII - XIX ${ }^{\mathrm{e}}$ siècles) // Les Français dans la vie intellectuelle et scientifique en Russie (XVIIIe-XXe siècles). Moskou : Olma Media Group, 2010. P. 87-105.

Liechtenhan F.-D. L'abbé Bignon, précurseur des relations scientifiques et culturelles avec la Russie // Les Français dans la vie intellectuelle et scientifique en Russie (XVIII $\mathrm{XX}^{\mathrm{e}}$ siècles). Moskou : Olma Media Group, 2010. P. 127-140.

Mémoires intéressantes envoyés [sic] par des missionnaires catholiques de Moscou au Pape sur les espérances de la prochaine réunion de l'Église russe à l'Église catholique // Monuments historiques de Russie. Monuments relatifs aux règnes d'Alexis Michaélowitch, Fedor III et Pierre le Grand czars de Russie, extraits des archives du Vatican et de Naples, par Augustin Theiner. Rome : Imprimerie du Vatican, 1859. P. 409 sq.

Mézin A. Correspondance des consuls de France à Saint-Pétersbourg, 1713-1792. Paris : Archives nationales, 2009.

Mézin A., Rjéoutski V. Les Français en Russie au siècle des Lumières. Ferney-Voltaire, Centre international d'Etude du XVIIIe siècle, 2011. Vol. 2. P. 483-486.

Pleyer $O$. Allerunterthänigste relation von dem jetzigen moscowitischen Regierungswesen, 1710 // Russland unter Peter dem Grossen / ed. by E. Herrmann. Leipzig, 1872. S. $133-135$.

Rambaud A. Recueil des Instructions données aux ambassadeurs et ministres de France // Mémoire pour servir à l'Instruction de... allant à Pétersbourg, 2 février 1714. Paris : Alcan, 1890. Vol. 1. P. 132-134.

Wittram R. Peter I. Czar und Kaiser. Göttingen : Vandenhoeck und Ruprecht, 1964. Bd. 2. S. 226-227.

\section{References}

Bély, L. (2007). L'Art de la paix en Europe, naissance de la diplomatie moderne, $X V I^{{ }^{e}-}$ $X V I I I^{e}$ siècles. Paris: PUF.

Demeulenaere-Douyère, C. (2010). L'Académie des Sciences de Paris ; un lieu privilégié des échanges scientifiques entre la France et la Russie (XVIII ${ }^{\mathrm{e}}-\mathrm{XIX}^{\mathrm{e}}$ siècles). 
In Les Français dans la vie intellectuelle et scientifique en Russie (XVIII ${ }^{\mathrm{e}}-\mathrm{XX}$ siècles) (pp. 87-105). Moskou: Olma Media Group.

Liechtenhan, F.-D. (2010). L'abbé Bignon, précurseur des relations scientifiques et culturelles avec la Russie. In Les Français dans la vie intellectuelle et scientifique en Russie (XVIII ${ }^{e}-X X^{e}$ siècles) (pp. 127-140). Moskou: Olma Media Group.

Lishtenan, F.-D. (Guzevich, I. D. \& Guzevich, D. Yu., transl.). (2011). «Esli Papa schitaet sebya nepogreshimy'm, to on, dolzhno by't', glupecz, a esli ne schitaet, to moshennik» (Petr Velikij) [If the Pope considers himself infallible, he must be a dolt, and if he does not, he must be a swindler]. In Kul'turny'e iniciativy' Petra Velikogo : materialy' II Mezhdunarodnogo kongressa petrovskih gorodov (pp. 58-62). Saint Petersburg: Evropejskij dom.

Mémoires intéressantes envoyés [sic] par des missionnaires catholiques de Moscou au Pape sur les espérances de la prochaine réunion de l'Église russe à l'Église catholique. (1859). In Monuments historiques de Russie. Monuments relatifs aux règnes d'Alexis Michaélowitch, Fedor III et Pierre le Grand czars de Russie, extraits des archives du Vatican et de Naples, par Augustin Theiner. Rome: Imprimerie du Vatican.

Mézin, A. (2009). Correspondance des consuls de France à Saint-Pétersbourg, 17131792. Paris: Archives nationales.

Mézin, A. \& Rjéoutski, V. (2011). Les Français en Russie au siècle des Lumières (Vol. 2, pp. 483-486). Ferney-Voltaire, Centre international d'Etude du XVIII ${ }^{\mathrm{e}}$ siècle.

Ozhero, K. (Tyan'shina, N. P., transl.). (2008). Uchrezhdenie pervogo konsul'stva Francii v Sankt-Peterburge v 1717 godu [Establishment of the first consulate of France in Saint Petersburg in 1717]. In P. P. Cherkasov (Ed.). Rossiya i Franciya. XVIII-XX veka. (Iss. 8, pp. 9-30). Moskow: Nauka.

Pleyer, O. (1872). Allerunterthänigste relation von dem jetzigen moscowitischen Regierungswesen, 1710. In E. Herrmann (Hrsg.) Russland unter Peter dem Grossen (pp. 133-135). Leipzig.

Rambaud, A. (1890). Recueil des Instructions données aux ambassadeurs et ministres de France. In Mémoire pour servir à l'Instruction de ... allant à Pétersbourg, 2 février 1714 (Vol. 1, pp. 132-134). Paris: Alcan.

Wittram, R. (1964). Peter I. Czar und Kaiser (Bd. 2, pp. 226-227). Göttingen: Vandenhoeck und Ruprecht.

The article was submitted on 12.05.2014

\author{
Франсин-Доминик Лиштенан \\ профессор, \\ Университет Париж-Сорбонна, \\ Национальный центр научных \\ исследований, \\ Париж, Франция \\ liechtenhan@sfr.fr
}

\author{
Francine-Dominique Liechtenhan \\ Professor, \\ Paris-Sorbonne University; \\ French National Centre for \\ Scientific Research, \\ Paris, France \\ liechtenhan@sfr.fr
}

